\title{
Faster recovery after radical cystectomy with alvimopan
}

New study data show that alvimopan-a $\mu$-opioid antagonist that competitively binds to, but doesn't activate, the $\mu$-opioid receptor in the gastrointestinal tractexpedites gastrointestinal recovery and improves early outcomes in patients undergoing radical cystectomy and receiving opioids, without increasing the risk of cardiovascular morbidity.

For this study, 280 patients with bladder cancer were randomized to receive alvimopan $(n=143)$ or placebo $(n=37)$, as well as postoperative intravenous patient-controlled opioids, after radical surgery at one of 21 highvolume centres in the USA. Patients who received alvimopan experienced quicker gastrointestinal recovery-a two-component end point combining first tolerance of food and first bowel movement-than those who received placebo (5.5 days vs 6.8 days), as well as shorter mean length of hospital stay (7.4 days vs 10.1 days), and fewer episodes of postoperative ileus (POI)-related morbidity ( $8.4 \%$ vs $29.1 \%$ ).

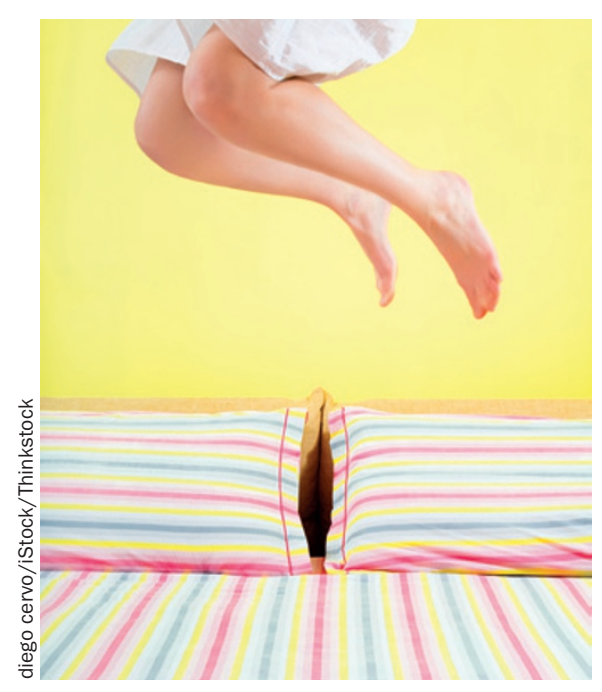

Aside from POI, the incidence of adverse events was comparable for the two treatment groups. Cardiovascular events occurred in just $8.4 \%$ of patients taking alvimopan compared with 15.3\% for placebo, suggesting that alvimopan does not pose a cardiovascular risk, as previously suggested. Indeed, alvimopan might offer protection against cardiac morbidity by reducing the risk of POI, which can cause fluid shifts into the bowel, leading to intravenous fluid replacement and loss of mobility.

"The evidence seems sufficiently compelling to recommend introduction of this agent into existing enhanced recovery pathways and evaluate its effect in related settings, such as robot-assisted surgery or programmes using epidural-based analgesia," concludes McGrath.

"POI is one of the most important and recalcitrant clinical issues known to delay recovery from radical cystectomy," says John McGrath, an independent expert in the field. "Historically, 30\% of patients undergoing this procedure experience POI, with the inherent delay in recovery and its associated morbidity."
Melanie Clyne

Original article Lee, C. T. et al. Alvimopan accelerates gastrointestinal recovery after radical cystectomy: a multicenter randomized placebo-controlled trial. Eur. Urol. doi:10.1016/j.eururo.2014.02.036 